\title{
Revisión Sistemática sobre Competencias de Autorregulación del Aprendizaje en Estudiantes Universitarios y Programas Intracurriculares para su Promoción
}

\author{
Fabiola M. Sáez ${ }^{(1)}$, Alejandro E. Díaz ${ }^{(2)}$, Ernesto Panadero(3) y Daniela V. Bruna ${ }^{(4)}$ \\ (1) Universidad Católica de la Santísima Concepción, Facultad de Educación, Departamento de \\ Fundamentos de la Pedagogía, Alonso de Ribera 2850, Concepción, Chile. (e-mail: fsaez@ucsc.cl) \\ (2) Universidad de Concepción, Facultad de Ciencias Sociales, Departamento de Psicología, Víctor Lamas \\ 1290, Concepción, Chile. (e-mail: adiazm@udec.cl) \\ (3) Universidad Autónoma de Madrid, Facultad de Psicología, Departamento de Psicología Evolutiva y de la \\ Educación, c/lvan Pavlov no6, Madrid, España. (e-mail: ernesto.panadero@uam.es) \\ (4) Centro de Investigación y Mejoramiento de la Educación (CIME), Universidad del Desarrollo, Chile. \\ (e-mail: dbrunaj@udd.cl)
}

Recibido Abr. 24, 2018; Aceptado Jun. 19, 2018; Versión final Jul. 25, 2018, Publicado Dic. 2018

\begin{abstract}
Resumen
Este trabajo tiene dos objetivos: (1) caracterizar investigaciones sobre competencias de autorregulación del aprendizaje y (2) describir características, limitaciones y orientaciones de programas intracurriculares para la promoción de la autorregulación del aprendizaje en estudiantes universitarios. El método utilizado es una revisión sistemática de artículos publicados entre los años 2010 y 2016, de tipo teórico, instrumental y empírico, sobre la promoción de la autorregulación del aprendizaje en el contexto de Educación Superior, en las bases de datos Web of Science (WOS), Scopus y SciELO. Los principales resultados son: caracterización, descripción, limitaciones y orientaciones de intervenciones para la promoción de la autorregulación del aprendizaje en Educación Superior. En conclusión, es necesario (1) desarrollar programas de tipo intracurricular para la promoción de competencias de autorregulación del aprendizaje en estudiantes universitarios y (2) orientar nuevas investigaciones centradas en la implicación de los profesores en este proceso.
\end{abstract}

\section{Systematic Review on Self- Regulated Learning Competencies in University Students and Intracurricular Programs for its Promotion}

\begin{abstract}
This paper has two aims: (1) to characterize the research done on self-regulated learning competencies and (2) to describe the characteristics, limitations and orientations of intra-curricular programs for the promotion of self-regulated learning in university students. The method is a systematic review of articles published between 2010 and 2016, of a theoretical, instrumental and empirical nature, on the promotion of self-regulation of learning in the context of Higher Education. These papers belong to the Web of Science (WOS), Scopus and SciELO databases. The main results are the characterization, description, limitations and orientations of interventions for the promotion of self-regulation of learning in Higher Education. In conclusion, it is necessary (1) to develop intracurricular programs for the promotion of self-regulated learning competencies in university students and (2) to guide new research focused on the involvement of teachers in this process.
\end{abstract}

Keywords: training programs; promotion; intracurricular; higher education 


\section{INTRODUCCIÓN}

El paso de la Enseñanza Media a la universidad constituye una etapa crítica, ya que este proceso de separación, transición e incorporación a un nuevo mundo social y académico, genera una línea de fractura entre el ciclo educativo altamente estructurado y controlado que existe en la Enseñanza Media y la cultura de aprendizaje independiente que se promueve en la Educación Superior, esto lleva a los estudiantes a experimentar dificultades académicas, fenómeno que ocurre a nivel internacional (Beaumont et al., 2016; Dörrenbächer y Perels, 2016; Elvira-Valdés y Pujol, 2012; Gale y Parker, 2014; Koivuniemi, Panadero, Malmberg, y Järvelä, 2017; Yau, Sun, y Fong, 2012). Además, la transición al nuevo sistema educativo de la universidad, evidencia una preparación insuficiente, con un déficit considerable en el desarrollo de estrategias de aprendizaje en los estudiantes, que no le permiten adquirir un compromiso constante y autónomo en el estudio (Hernández, Rosário y Cuesta, 2010). Se ha demostrado que un desarrollo académico inadecuado durante los primeros años de experiencia universitaria es explicado entre otras variables, por la deficiencia de empleo de estrategias de autorregulación del aprendizaje (Cazan, 2012; Tuckman y Kennedy, 2011). La incapacidad de estudiantes novatos para alcanzar las exigencias académicas implica en ellos un factor causal significativo en el fracaso académico y abandono precoz (Miranda y Guzmán, 2017; Stoten, 2015). Ambos fenómenos son preocupaciones y desafíos de las universidades (Medrano, Galleano, Galera, y Del Valle., 2010). La clave es cómo ayudar a los estudiantes a afrontar su aprendizaje de forma intencional, autónoma y efectiva, proceso denominado "autorregulación del aprendizaje" (Panadero y Alonso-Tapia, 2014).

La autorregulación del aprendizaje o Self Regulated Learning por sus siglas en inglés SRL, es definida como un proceso autodirigido donde los estudiantes transforman sus habilidades mentales en habilidades académicas, autogenerando pensamientos, sentimientos y comportamientos que están orientados a la consecución de metas (Zimmerman, 2002). Este proceso de aprendizaje permite reunir los diferentes elementos que ponen a los estudiantes en el centro de su propio aprendizaje (Stoten, 2015). Los estudiantes universitarios con éxito se identifican por sus habilidades y capacidades para orientar su comportamiento a través de un conjunto de estrategias de disposición al estudio, cognitivas y metacognitivas (Pérez et al., 2011). Específicamente, los alumnos autorregulados se caracterizan por su iniciativa, control, perseverancia y dominio de estrategias. Estas cualidades se relacionan con mejores resultados académicos (Cerezo et al., 2010). Los procesos de autorregulación del aprendizaje en estudiantes universitarios son fundamentales para alcanzar el manejo, el control y el monitoreo de metas, estrategias, y motivaciones que aparecen en la realización de las tareas académicas (Cohen, 2012; Garello y Rinaudo, 2012). Por tanto, desempeñan un papel determinante en el éxito académico de los estudiantes favoreciendo la consecución de logros académicos y previniendo el fracaso académico (Schober et al., 2015). La autorregulación es relevante para en la Educación Superior respecto del comportamiento de los alumnos y el rendimiento académico (Bembenutty, 2011).

Una de las metas centrales de las universidades en el siglo XXI es lograr que los estudiantes controlen su proceso de estudio y adquieran competencias que les sean útiles dentro y fuera de las aulas, proporcionando una formación que garantice su ajuste a la sociedad del conocimiento, cuyo eje central gira en torno al aprendizaje autónomo y a lo largo de la vida, es decir, en torno al aprendizaje autorregulado (Dresel et al., 2015; Schober et al., 2015). El desarrollo de competencias es recomendado realizarlo en los primeros años de universidad (Cassidy, 2011; Cerezo et al., 2011). Considerando estos argumentos, está justificada la necesidad de poner en marcha programas para avanzar en la promoción de estrategias de autorregulación en los alumnos, persiguiendo mayor eficacia e innovación en las intervenciones. Se destaca la necesidad de diseñar y desarrollar más intervenciones que permitan mejorar los procedimientos de estudio de los estudiantes universitarios, capacitándolos para autorregular su propio aprendizaje (Sitzmann y Ely, 2011). El desafío está en enfocarse en el aprendizaje de competencias instrumentales sobre aplicación y puesta en práctica habitual de procedimientos más que un conocimiento declarativo sobre ellas (Pérez et al., 2013).

Los programas desarrollados en contexto universitario son: (a) cursos de capacitación llamados "aprender a aprender", para que los estudiantes identifiquen y utilicen estrategias apropiadas a las diferentes tareas y situaciones de aprendizaje, esta orientación promueve la transferencia de los aprendizajes a otros contextos; (b) intervenciones puntuales, cursos de verano o programas puente entre dos ciclos de estudio, orientados sobre la lógica de cubrir lagunas, focalizada en potenciar aspectos estratégicos aislados; (c) inclusión curricular, este método se utiliza para ayudar a los alumnos a desarrollar estrategias de aprendizaje efectivas, donde el docente instruye a sus estudiantes en las cuestiones motivacionales y estrategias cognitivas relativas a su área de contenido (Díaz, Pérez, González-Pienda, y Núñez, 2017). Un programa tiene la característica de intracurricular cuando es realizado mediante actividades propias del plan de estudio (Pérez et al., 2011). 
Es decir, los programas para la promoción de competencias de autorregulación del aprendizaje pueden ser clasificados en programas extracurriculares (a y b) y programas intracurriculares (c).

De las dos clasificaciones expuestas las más escasas en la literatura pero que han mostrado mayor efectividad son los programas de intervención intracurricular (Cerezo et al., 2011). Parece conveniente desarrollar trabajos de intervención que muestren resultados empíricos y que además estas sean parte del currículum, pues aún se encuentra en un estado inicial de desarrollo (Díaz et al., 2017; Rosário et al., 2014). En Educación Superior, integrar o difundir estrategias de autorregulación del aprendizaje en el desarrollo de los contenidos curriculares de cada asignatura del plan de estudios es una necesidad (Carbonero y Navarro, 2006). Ya se ha dejado de lado el paradigma relacionado con completar lagunas de contenidos, siendo sustituido por la promoción de competencias para un aprendizaje autónomo (Rosário et al., 2007). Un desafío sería implementar programas de mejora de las estrategias de autorregulación con el fin de facilitar la transición de la Enseñanza Media a la Educación Superior en el currículo universitario de primer año (Beaumont, Canning, y Moscrop, 2016; Rosário, Fuentes, Beuchat, y Ramaciotti, 2016; Vassallo, 2011).

Un desafío importante, es facilitar en el aula el empleo de competencias de autorregulación del aprendizaje (Cassidy, 2011; Díaz et al., 2017; Rosário et al., 2016). Es de decir, enseñadas junto con los contenidos curriculares de cada asignatura (Roux y Anzures, 2015). Las iniciativas de promoción de estrategias de autorregulación en el contexto universitario se instalan de manera poco sistemática y aisladamente como programas de nivelación y/o remediales (Navarrete et al., 2013; Soto, 2016). Aunque se han desarrollado programas de intervención promoviendo procesos de autorregulación, los artículos publicados sobre programas intracurriculares son escasos, y pocos estudios muestran resultados empíricos (Beaumont et al., 2016; Cerezo et al., 2010; Dörrenbächer y Perels, 2016). Este tipo de intervenciones son efectivas, pero se encuentra en un estado inicial de desarrollo (Rosário et al., 2014). En el desarrollo de las tareas propias del currículum de su disciplina, los docentes pueden ofrecer modelos a sus estudiantes y discutir con ellos la aplicación de estrategias de autorregulación a situaciones cotidianas de enseñanzaaprendizaje, facilitando primero su aplicación supervisada y, luego la aplicación autónoma (Díaz et al., 2017; Pérez y Díaz, 2013; Roux y Anzures, 2015). Específicamente a través del entrenamiento intracurricular de estrategias de autorregulación del aprendizaje y su posterior uso, se contribuye a la formación integral de las nuevas generaciones de universitarios (Carbonero, Román, y Ferrer, 2013).

No se ha encontrado una sistematización sobre las intervenciones y la promoción de la autorregulación del aprendizaje en estudiantes universitarios en el período 2010-2016 de las bases de datos WOS, Scopus y SciELO, sólo una sistematización de los artículos publicados en la base Scielo entre los años 2001 y 2011 donde el tema principal era la autorregulación del aprendizaje (Rosário et al., 2014). En la Educación Superior es importante conocer la producción que se ha realizado sobre la autorregulación del aprendizaje, ya que esto permite direccionar los esfuerzos en la mejora de esta competencia, a través del desarrollo de diversas propuestas e intervenciones.

Es un reto en Educación Superior proponer modelos, programas o situaciones de aprendizaje para que el estudiante tenga éxito académico, la solución que ha encontrado la investigación es el entrenamiento de estrategias de autorregulación del aprendizaje como elemento determinante en el proceso de aprendizaje (Huie et al., 2014). La presente investigación se plantea como objetivo general sistematizar los estudios científicos sobre la promoción y desarrollo de la autorregulación del aprendizaje, para entregar antecedentes a los investigadores que tengan por objetivo desarrollar programas o intervenciones de mejora de las competencias de autorregulación en Educación Superior.

\section{METODOLOGÍA}

El método aplicado en el presente trabajo, consistió en una revisión sistemática de artículos indizados en las principales bases de datos dentro del marco académico y científico de las ciencias psicológicas, éstas fueron: Scopus, Web of Science y, SciELO.

\section{Proceso de selección de los artículos}

Los criterios generales de elegibilidad de los artículos se basaron en las siguientes características: (1) publicación de tipo teórica o empírica que aporte antecedentes a la promoción de la autorregulación del aprendizaje en contexto de Educación Superior; (2) los participantes son estudiantes universitarios; y (3) los idiomas de búsqueda fueron español e inglés. Se busca conocer lo que se ha hecho para el desarrollo de competencias de autorregulación en estudiantes universitarios. Las fuentes de búsqueda estuvieron limitadas a las publicaciones de las bases de datos WOS, Scopus y SciELO comprendida en el período de tiempo desde el año 2010 al 2016. 
La última fecha de búsqueda se llevó a cabo el 20 de septiembre de 2016. La revisión sistemática, siguió una estrategia de búsqueda específica para cada base de datos (WOS, Scopus y SciELO), basada en la refinación de criterios con el fin de que esta se pueda reproducir. En el caso de la base de datos SciELO, el descriptor utilizado fue menos específico por las características de esta base de datos respecto de los procedimientos de búsqueda. La muestra seleccionada es de 30 artículos, que cumplen con los criterios de inclusión. Estos se pueden revisar en la tabla 1.

Tabla 1: Criterios específicos de elegibilidad para la búsqueda sistemática de artículos

\begin{tabular}{|c|c|c|c|}
\hline Refinación de búsqueda & Web of Science (WOS) & Scopus & ScIELO \\
\hline Nombre de los descriptores & \multicolumn{2}{|c|}{$\begin{array}{l}\text { (Increasing "self regulated learning") OR (Promote "self } \\
\text { regulated learning") OR } \\
\text { (Fostering "self regulated learning") OR } \\
\text { (Promotion "self regulated learning") OR (Encouracing "self } \\
\text { regulated learning") OR Intervention "self regulated learning" } \\
\text { OR (Program "self regulated learning") OR (Training "self } \\
\text { regulated learning") AND (College Student) And (Higher } \\
\text { Education }\end{array}$} & $\begin{array}{l}\text { "Autorregulación } \\
\text { aprendizaje" }\end{array}$ \\
\hline $\begin{array}{l}\text { Ubicación de descriptores } \\
\text { OR }\end{array}$ & En título & $\begin{array}{l}\text { Artículo, título, resumen o } \\
\text { palabras claves }\end{array}$ & Todos los índices \\
\hline $\begin{array}{l}\text { Ubicación de descriptores } \\
\text { AND }\end{array}$ & En Tema & $\begin{array}{l}\text { Artículo, título, resumen o } \\
\text { palabras claves }\end{array}$ & - \\
\hline Período de tiempo & $2010-2016$ & $2010-2016$ & $2010-2016$ \\
\hline Áreas de investigación & Todas & Todas & Todas \\
\hline Tipo de documento & Artículo & Artículo & Artículo \\
\hline Idioma & Español e inglés & Español e inglés & Español e inglés \\
\hline Resultados de búsqueda & $\begin{array}{lr}\text { Veinticinco } & \text { artículos } \\
\text { encontrados. } & \text { Cumplen } \\
\text { criterio de inclusión doce } \\
\text { artículos. }\end{array}$ & $\begin{array}{l}\text { Siete artículos encontrados. } \\
\text { Cumplen criterio de inclusión } \\
\text { seis artículos. }\end{array}$ & $\begin{array}{lr}\text { Dieciséis } & \text { artículos } \\
\text { encontrados. } & \text { Cumplen } \\
\text { criterio de inclusión doce } \\
\text { artículos. }\end{array}$ \\
\hline
\end{tabular}

Proceso de análisis de las características de los artículos seleccionados

Para registrar los datos se utilizó un protocolo que permitió organizar las características de cada artículo. El protocolo para el análisis de los artículos recogía información de los siguientes campos: fuente, país, objetivos, tamaño de la muestra, diseño del estudio, y objetivo (Tabla 2). La clasificación del diseño de investigación de los artículos se basó en cuatro tipos: (a) investigación teórica, (b) investigación instrumental, (c) investigación metodológica e, (d) investigación empírica (Ato, López-García y Benavente, 2013).

\section{Proceso de análisis de las características de los programas de intervención}

Se utilizó un protocolo que permitió describir cada programa de intervención, recogiendo información de los siguientes campos: (1) fuente, (2) nombre del programa (3) variable independiente, (4) variables dependientes, (5) tipo de instrumento y (7) tipo de intervención. (Tabla 3).

\section{Proceso de descripción, principales limitaciones y orientaciones de las intervenciones}

Para la descripción de los programas intracurriculares se consideró: en qué consistió, método, resultados y conclusiones. Las limitaciones de los estudios se analizan respecto del método y procedimiento descrito, y las orientaciones corresponden a sugerencias declaradas por los autores para futuras propuestas de desarrollo y aplicación de programas de intervención. 


\section{RESULTADOS}

Se presentan los resultados de acuerdo a tres análisis. El primero caracteriza todos los artículos seleccionados en este estudio. El segundo análisis entrega antecedentes de las principales características de los programas de intervención. Finalmente un tercer análisis describe las experiencias de programas de intervención de tipo intracurricular, que tienen por objetivo promover competencias de la autorregulación del aprendizaje, indicando limitaciones y orientaciones para futuros trabajos en esta línea de investigación.

\section{Resultados de las características de los artículos seleccionados}

De acuerdo a los resultados de los artículos encontrados, que cumplen con los criterios de inclusión en este estudio, los países donde se desarrollaron las investigaciones corresponden a: 11 en España, 5 en Chile, 3 en Estados Unidos, 3 en Reino Unido, 2 en Portugal, 1 Taiwán, 1 en China, 1 en Alemania, 1 en Mozambique, 1 en Canadá, 1 en Malasia, 1 en El Salvador, 1 en Argentina, 1 en Brasil y 1 en México.

El tamaño de la muestra varía entre 15 estudiantes y 2.429 estudiantes. Respecto del diseño del estudio, 21 artículos presentan resultados empíricos, 8 son teóricos y 1 es instrumental. Sin embargo, 13 artículos son experiencias de programas de intervención, y específicamente 8 de ellos son intervenciones intracurriculares.

Los objetivos de todos los artículos están relacionados a la promoción de la autorregulación del aprendizaje. Principalmente se plantean objetivos en relación a 7 temas: (1) evaluación de la eficacia de programas de intervención, (2) comparación de la eficacia de programas, (3) descripción de actividades, prácticas o experiencias que fomentan la autorregulación del aprendizaje, (4) relación o capacidad predictiva de determinadas variables con el uso de estrategias de autorregulación, (5) análisis de modelos de autorregulación, (6) formas y procedimientos para la evaluación de la autorregulación, y (7) papel de los medios tecnológicos y la autorregulación.

\section{Resultado del proceso de análisis de las características de los programas de intervención}

En la tercera columna de la tabla 3, se puede observar el nombre de los programas para el fomento de la autorregulación del aprendizaje en estudiantes universitarios. Si bien se analizaron 13 estudios de intervención, de estos, diez eran programas diferentes:

(1) andamios externos para fomentar SRL;

(2) diario de aprendizaje Basado en el modelo de Zimmerman;

(3) docencia para promover SRL;

(4 a) Training for an Autonomous Learning (TRAL), entrenamiento para un aprendizaje autónomo (4 b) e

TRAL, entrenamiento para un aprendizaje autónomo en plataforma electrónica,

(5) Cartas de Gervasio;

(6) Linguafolio;

(7) Dialogic Feedback Cycle (DFC), Ciclo de Retroalimentación Dialógico;

(8) Coaching Educativo;

(9) Enfoque de enseñanza autorregulada;

(10) Programa instruccional basado en el modelo de Pintrich

Las variables independientes de los estudios son los respectivos programas de intervención, y las variables dependientes de los diferentes trabajos son: (1) participación en la realización de tareas de aprendizaje en línea, (2) uso de estrategias de autorregulación del aprendizaje, (3) rendimiento académico, (4) fases de autorregulación del aprendizaje, (5) estudiantes que aprueban el curso, (6) satisfacción del material utilizado en el programa, (7) satisfacción de las características y utilidad del programa, (8) complejidad estructural de las respuestas de los alumnos a una tarea, (9) instrumentalidad percibida del uso de estrategias autorregulación del aprendizaje, (10) creencias docentes en relación a la teoría en aula centrada en el estudiante, (11) percepción de la comprensión de las tareas de evaluación, (15) percepción de la comprensión de criterios de evaluación, (16) Autominusvalía académica, y (17) adquisición de conceptos científicos.

Los instrumentos de medición de las variables de autorregulación del aprendizaje, son de tipo autorreporte, a excepción de una investigación donde se utilizó medición procesual de las variables en estudio. Finalmente del análisis de las 13 investigaciones que aplicaron programas de intervención, la mayoría es de tipo intracurricular, específicamente 8 de ellas. Estas intervenciones son propuestas que buscan desarrollar competencias de autorregulación en el propio proceso de enseñanza de los contenidos específicos de un dominio de aprendizaje. De las investigaciones de tipo extracurricular, sólo se encontraron 5 experiencias. Estos son propuestas de cursos, seminarios o talleres, y no están relacionados con un dominio específico. 


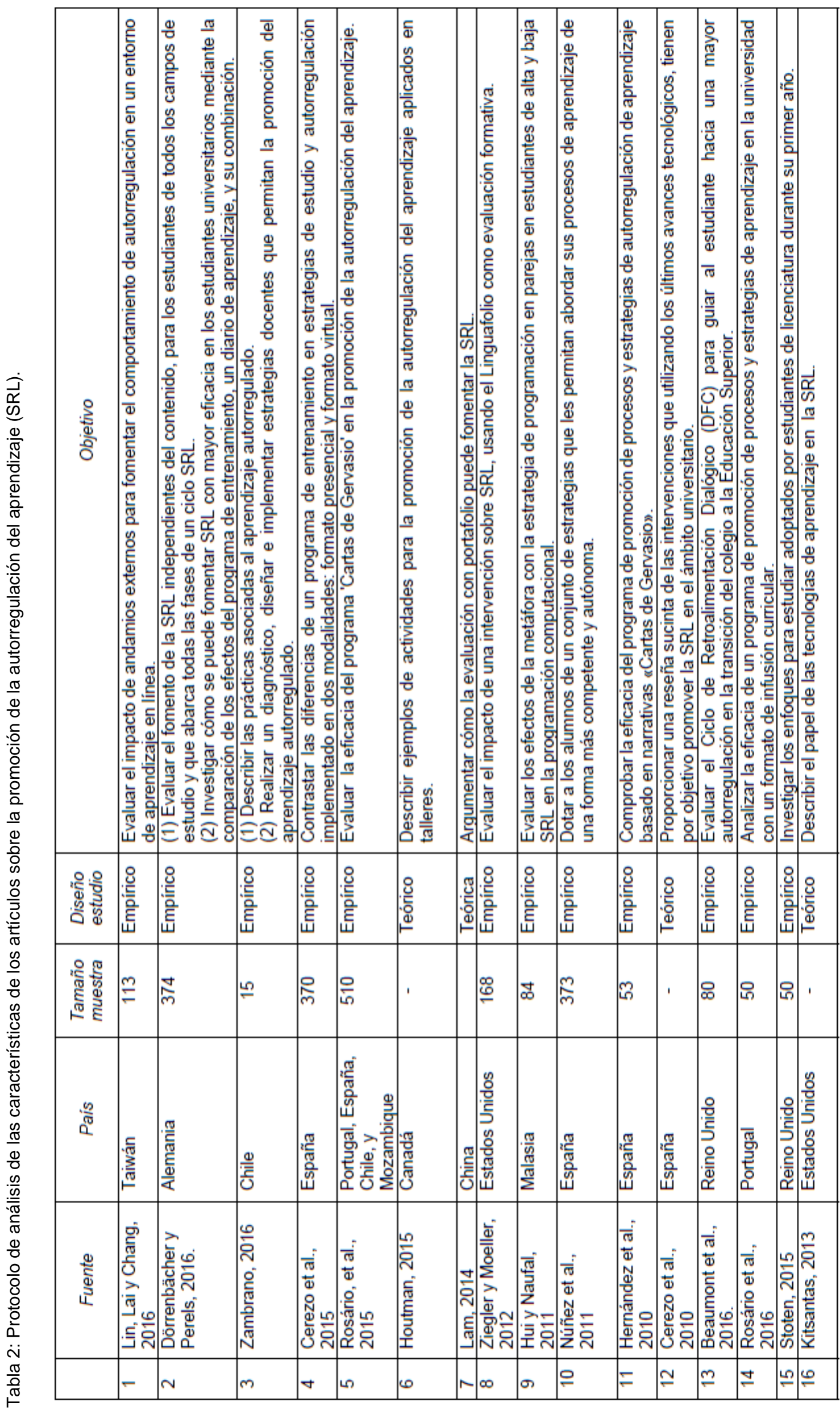




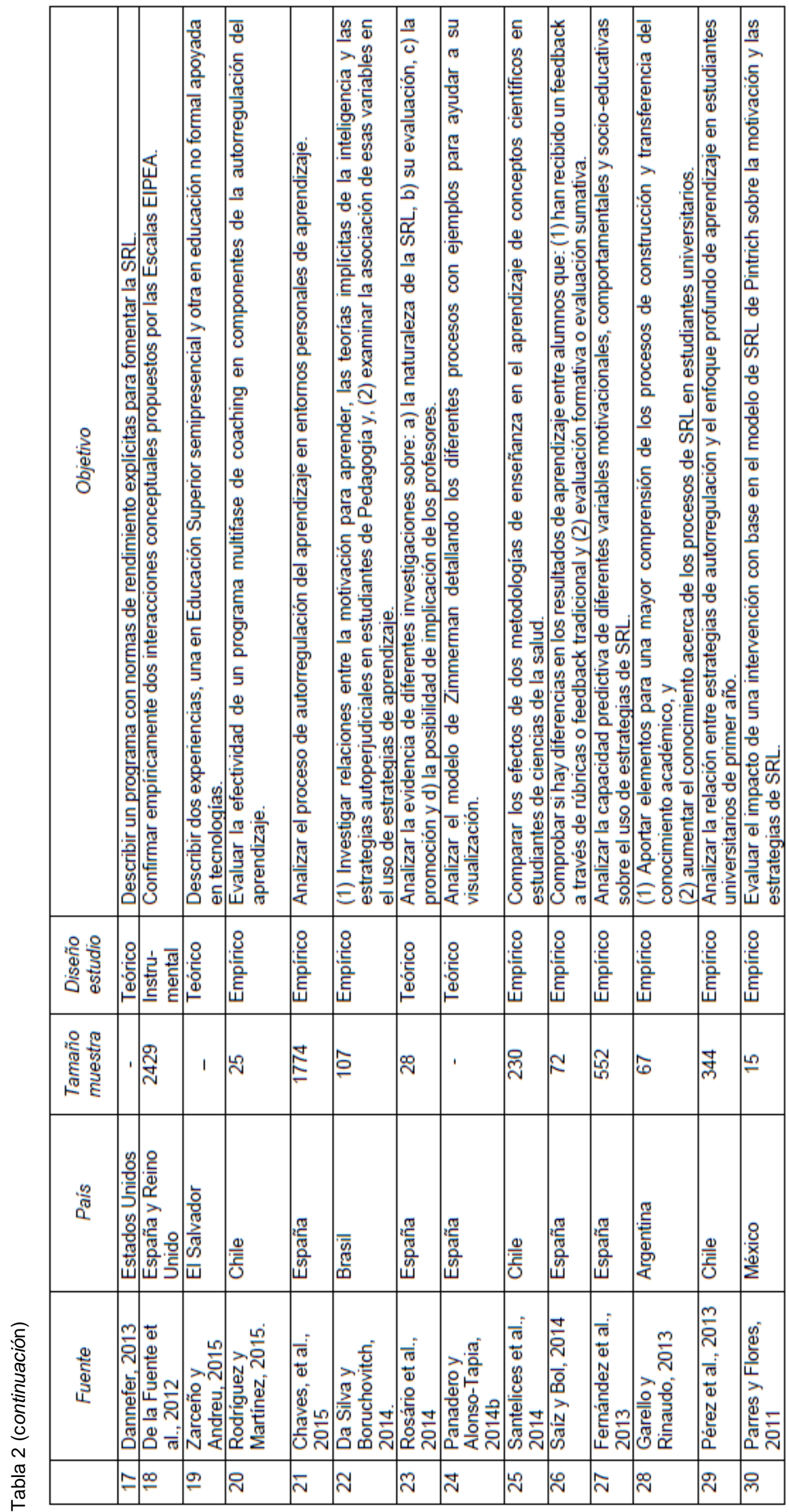




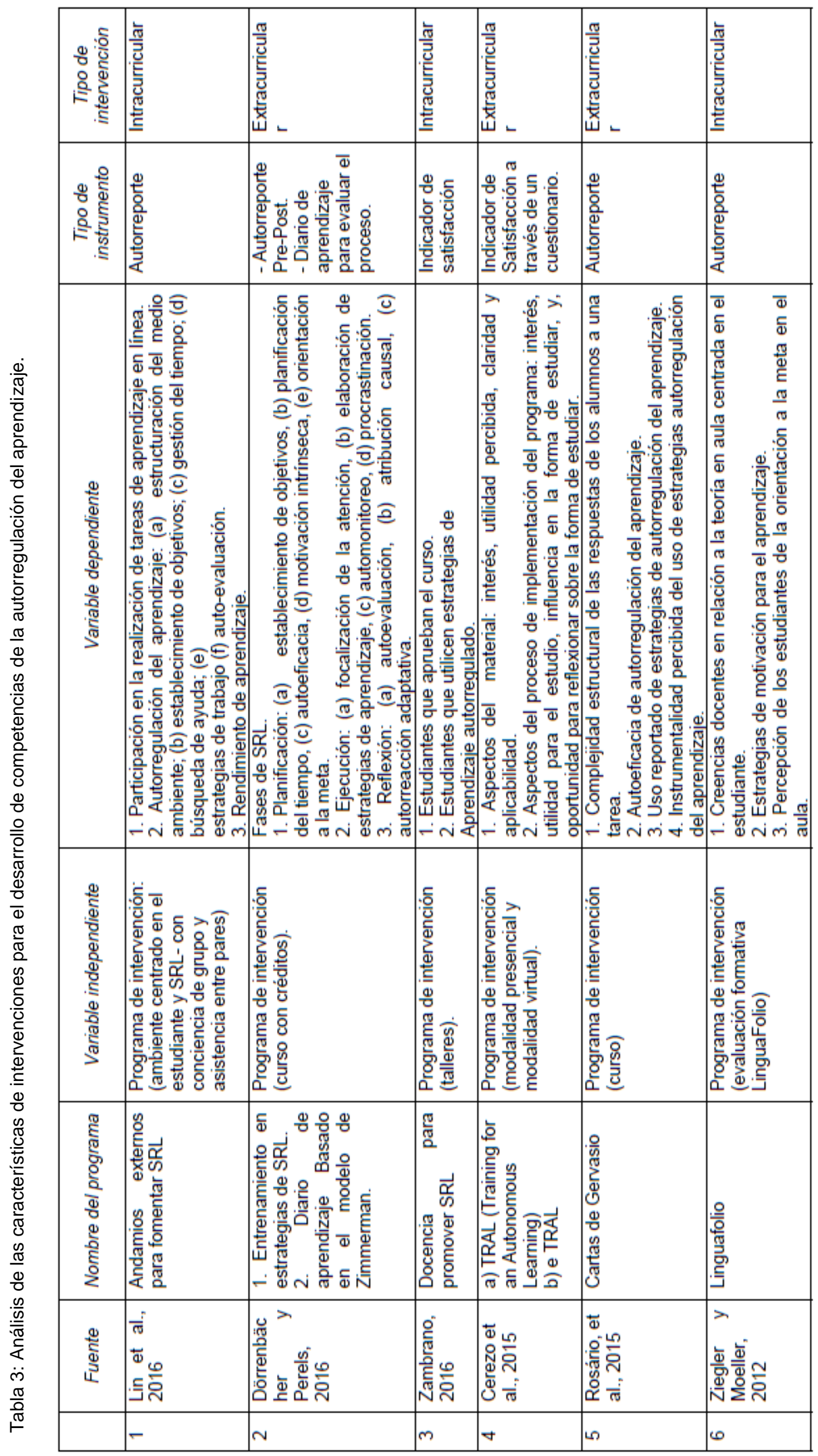




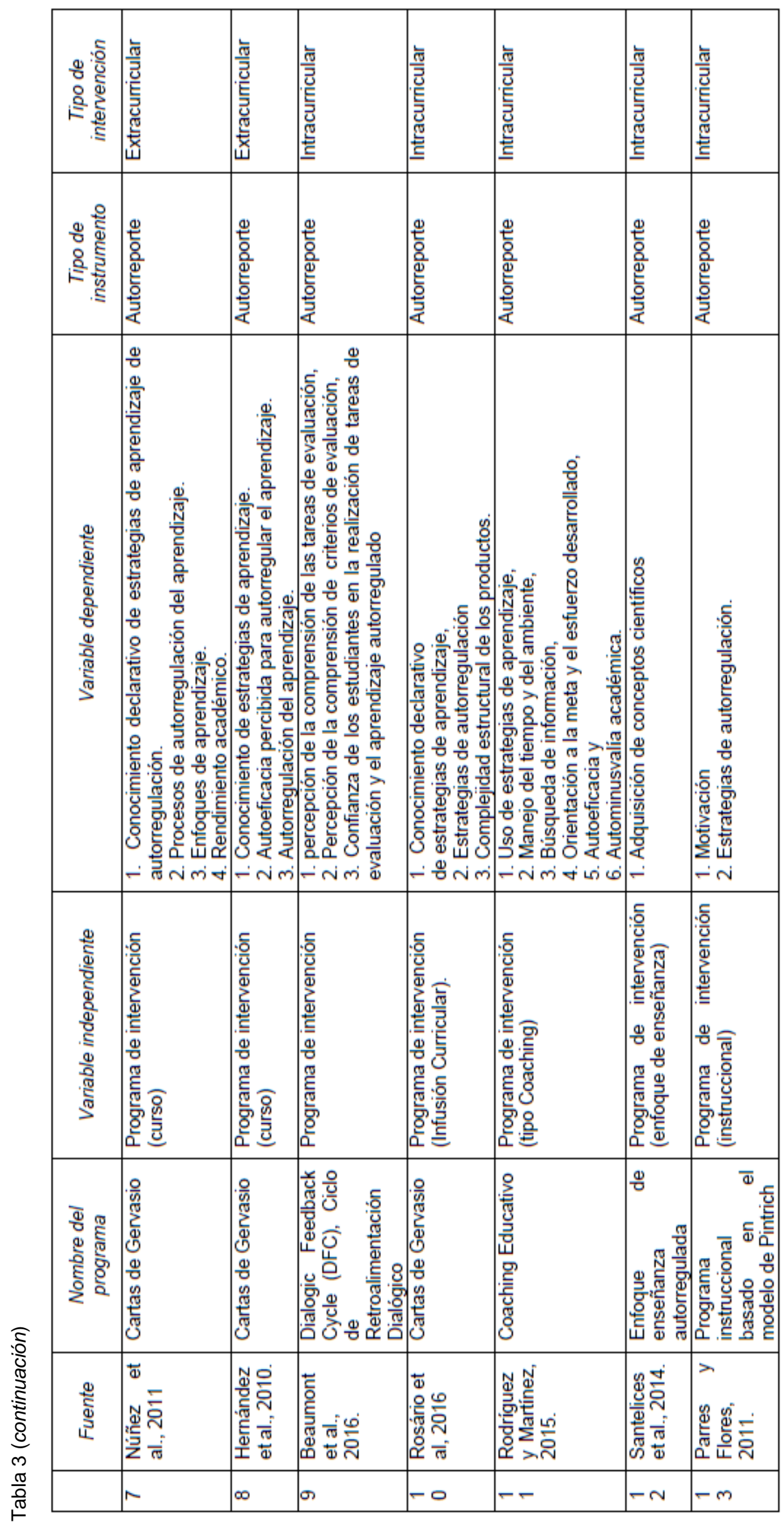


Resultados del proceso de análisis de las intervenciones de tipo intracurricular

Los resultados de este análisis se presentan en 3 aspectos diferentes: (1) descripción, (2) limitaciones, y (3) orientaciones de las intervenciones.

Descripción de las intervenciones de tipo intracurricular. Específicamente se describen 4 elementos de las intervenciones de tipo intracurricular: (1) en qué consistió el programa, (2) método, (3) resultados, y (4) conclusiones.

A) Fomentar el aprendizaje autorregulado utilizando la conciencia de grupo y ayuda como andamios externos (Lin et al., 2016). Consistió en la aplicación de dos formas de promoción de la SRL: (1) SRL-T (ambiente centrado en el estudiante y sin conciencia de grupo y asistencia entre pares); y (2) SRL-GAPA (SRL- con conciencia de grupo y asistencia entre pares). La principal diferencia es que en SRL-GAPA los estudiantes podían motivar y ayudar a los demás dentro de su propio grupo proporcionando respuestas, aunque no siempre correctas. En SRL-T los estudiantes reciben respuestas correctas si se responde incorrectamente. El método de intervención fue en dos cursos, en uno se aplicó SRL-T y en el otro SRL-GAPA con las siguientes características en común: duró 2 horas por 8 semanas, cada fase del ciclo de SRL se capacitó en 1 semana, pasaron por seis ciclos del proceso de formación SRL de Zimmerman, 6 semanas en total, para cada capítulo correspondieron evaluaciones sobre preguntas de selección múltiple basadas en las materias que se enseñaban, el mismo profesor enseñó a ambos cursos con el mismo material. Los resultados muestran diferencias en las variables: (1) participación, (2) comportamiento de SRL, y (3) resultados de aprendizaje en la clase SRL-GAPA, a diferencia de la clase de SRL-T. Las conclusiones del estudio indican que SRL-GAPA benefició más a los estudiantes con un bajo nivel SRL que aquellos con un alto nivel SRL. La conciencia de grupo estimula la colaboración entre pares y ofrece a los estudiantes, particularmente aquellos con un nivel bajo de SRL, emular modelos de conducta.

B) Autoeficacia, Prácticas de Aprendizaje Autorregulado y Docencia para fomentar el Aprendizaje Autorregulado en un Curso de Ingeniería de Software (Zambrano, 2016). Consistió en modelar una estrategia docente con técnicas para fomentar el aprendizaje autorregulado con un plan de planificación, monitorización y evaluación del estudio en la asignatura de ingeniería de software. Se aplicó una matriz CQA (C: ¿Qué conozco del tema?; Q: ¿Qué quiero aprender?; A: ¿qué aprendí?). El método contempló 16 clases de la asignatura Ingeniería de Software, donde semana por medio se implementó de forma intracurricular un taller (8 clases). De los 90 minutos que dura la clase, 20 minutos se utilizaron para la intervención donde los 10 minutos iniciales se aplican las letras $C$ y $Q$ de la matriz, y los 10 minutos finales la letra $A$ de la matriz. Los resultados muestran: (1) más del $75 \%$ de los estudiantes aprueban el curso y, (2) más del $75 \%$ de los estudiantes utilizan estrategias de aprendizaje para aprender autónomamente. El estudio concluye que los estudiantes no organizan, ni planifican su proceso de estudio. Tampoco existe una forma explícita ni clara de monitorear su proceso de aprendizaje. El diseño de la intervención permitió a los estudiantes instruirse en técnicas de estudio para la autonomía y organizar la clase en un formato de aprendizaje activo.

C) Aumentando el Aprendizaje Autorregulado a través del Linguafolio (Ziegler y Moeller, 2012). Consistió en la aplicación de un Linguafolio, definido como una colección sistemática de trabajos desarrollados por los alumnos en una asignatura en particular, el que es analizado para mostrar el progreso en el tiempo con respecto a los objetivos de instrucción. El método fue un semestre de duración, 102 clases. Se realizó en 9 cursos de Español y 3 de Francés. Español sirvió de grupo experimental $(\mathrm{N}=127)$ y Francés de grupo control $(\mathrm{N}=41)$. Los resultados mostraron diferencias estadísticamente significativas en: orientación a la meta, valor a la tarea, y creencias del instructor sobre la eficacia del Linguafolio. Se concluye que el Linguafolio es efectivo para aumentar la autorregulación del aprendizaje.

D) Facilitar la transición de la escuela a la Educación Superior: desarrollo del aprendizaje autorregulado como andamio a través de un enfoque dialógico de retroalimentación (Beaumont et al., 2016). Consistió en un conjunto estructurado de actividades de orientación como medio de inducción prolongado en los procesos de evaluación en Educación Superior basadas en el ciclo de retroalimentación Dialogic (DFC) que abarca los principios de retroalimentación como el diálogo, haciendo hincapié en la orientación al comienzo y durante una tarea en lugar de retroalimentación sumativa escrita. Específicamente las actividades fueron: orientación en la etapa preparatoria, orientación en la etapa misma de la tarea, y retroalimentación de la ejecución. El método fueron actividades aplicadas en el módulo Análisis de Sistemas de primer año de la carrera Licenciatura en Informática. Los resultados mostraron mejoras estadísticamente significativas $(p<0,05)$ en: percepción de la comprensión de las tareas de evaluación, percepción de la comprensión de criterios de evaluación, y confianza de los estudiantes en la realización de tareas de evaluación y el aprendizaje autorregulado. Se concluye que los estudiantes concuerdan que la retroalimentación fue oportuna, ayudó a su aprendizaje y la utilizaron para preparar la siguiente tarea; los estudiantes estaban más dispuestos a pedir ayuda a los tutores; los estudiantes ganaron confianza para planificar, investigar y aplicar los conocimientos 
de una manera más independiente; la adopción del DFC como modelo ha sido eficaz en desarrollar la SRL; los mejores niveles de confianza muestran una transición más exitosa a la Educación Superior.

E) Autorregulación del aprendizaje en una clase de la universidad: un enfoque de infusión curricular (Rosário et al., 2016). Consistió en un conjunto de cartas escritas por un estudiante de 1ำ (Gervasio) sobre sus experiencias académicas en la universidad. El método contempló 15 semanas (90 minutos por modulo semanal) en el contexto de una asignatura semestral. Las cartas se trabajaron en 12 sesiones (las otras 3 sesiones dedicadas a la administración de los instrumentos) y se siguió el siguiente diseño: 1) la carta fue leída y se analizó el contenido de forma individual (15 minutos); 2) los estudiantes comentaron el contenido, respondieron a preguntas de la docente y compartieron reflexiones (30 minutos); 3) los estudiantes participaron en talleres grupales e individuales aplicando las estrategias (30 minutos); 4) los alumnos extrajeron conclusiones, explicitando estrategias adquiridas en la sesión y transfiriéndolas a otras situaciones (15 minutos). Los resultados evidenciaron diferencias estadísticamente significativas entre el grupo experimental y control después de la intervención en: conocimiento declarativo de estrategias de aprendizaje, estrategias de autorregulación del aprendizaje, y complejidad estructural de los productos. Se concluye que el programa fue efectivo en la medida que, después del final del mismo, los sujetos del grupo experimental, en comparación con los del control, mostraron un mayor conocimiento de estrategias de autorregulación.

F) Efectividad del coaching grupal sobre el desarrollo de la autorregulación del aprendizaje en estudiantes de ingeniería (Rodríguez y Martínez, 2015). Consistió en ayudar a los estudiantes a aprender en lugar de enseñarles, guiándolos hacia sus metas en términos de conocimientos, habilidades y emociones. Para lograr un Coaching Educativo, el profesor aplica una metodología de trabajo precisa y acordada con los alumnos. Debe establecer relaciones empáticas y armoniosas con sus coachees (estudiantes bajo su mentoría), generando un clima que facilite el logro de objetivos. El método contempló cuatro meses durante un semestre académico. Las sesiones fueron de 90 minutos, con una frecuencia semanal. Los resultados, a excepción de orientación a la meta, tuvo un aumento estadísticamente significativo en las medias de todos los componentes de SRL: manejo del ambiente y la conducta, uso de estrategias de aprendizaje, búsqueda y aprendizaje de la información, estrategias de autominusvalía académica, y autoeficacia. Cualitativamente aumentó: calidad de las tareas escolares realizadas, mejora en su disposición al aprendizaje, aumento en el entusiasmo por aprender. El estudio concluye que el Programa de Coaching permitió el desarrollo de la competencia de autorregulación del aprendizaje.

G) Efecto del enfoque de autorregulación del aprendizaje en la enseñanza de conceptos científicos en estudiantes universitarios en ciencias de la salud (Santelices et al., 2014). Consistió en la comparación de dos metodologías de enseñanza (tradicional y autorregulada), sobre la adquisición de conceptos científicos. El método fue aplicar las dos metodologías en un curso con una semana de duración y un total de 40 horas pedagógicas (20 horas enseñanza tradicional, y 20 horas metodología de autorregulación). La muestra estaba compuesta por 137 estudiantes de primer año de medicina, 33 de kinesiología y 48 de enfermería.Los resultados mostraron diferencias estadísticamente significativas en los puntajes obtenidos en los post test, cuyo objetivo fue evaluar el aprendizaje de conceptos científicos claves, dependiendo de la metodología utilizada. El estudio concluyó que la metodología autorregulada favorece la adquisición de conceptos científicos en los cursos iniciales de ciencias de la salud más que la metodología tradicional.

H) Experiencia educativa en arte visual diseñada bajo un modelo de autorregulación del aprendizaje con estudiantes universitarios (Parres y Flores, 2011). Consistió en el diseño, desarrollo y evaluación de una situación de enseñanza considerando el modelo de Pintrich (2000) y enfocada a la enseñanza de las Artes Visuales. Se elaboró un manual sobre las estrategias de aprendizaje que el profesor debía promover durante las sesiones del taller. Se propusieron formas específicas de participación para el profesor y los estudiantes, para propiciar niveles cada vez más complejos de autorregulación y autonomía en la realización de la obra artística. El método aplicado fue el desarrollo de un taller de Artes Visuales (TAV), con 62 sesiones de 3 horas cada una, dos veces por semana; 30 sesiones para cinco unidades temáticas de dibujo y 32 para cinco unidades temáticas de escultura. Participaron de forma voluntaria 15 estudiantes (10 mujeres y 5 hombres). Los resultados encontraron diferencias estadísticamente significativas en orientación intrínseca a la meta, autoeficacia para el aprendizaje, ansiedad, autorregulación metacognitiva y búsqueda de ayuda. El estudio concluye que la programación de la enseñanza artística sustentada en el modelo de Paul Pintrich (2000), propicia un ciclo de autorregulación.

\section{Limitaciones de las intervenciones de tipo intracurricular}

En la presente investigación se seleccionaron 30 artículos que cumplieron con los criterios de inclusión, 21 artículos presentan resultados empíricos. Sin embargo, 13 artículos son experiencias de programas de intervención, y específicamente 8 de ellos son intervenciones intracurriculares. Además de ser escasas las experiencias encontradas en la literatura sobre el fomento de la autorregulación del aprendizaje, estas investigaciones presentan limitaciones en aspectos como el diseño, método y/o procedimientos. 
Respecto de las limitaciones asociadas principalmente al diseño, en el estudio de Lin et al. (2016), al mismo tiempo utilizan dos modalidades para mejorar la eficacia de la formación SRL (conciencia de grupo y asistencia entre pares), impidiendo determinar qué modalidad es el principal contribuyente a los resultados. No se describe aspectos importantes de los participantes (carrera que pertenecen, asignatura intervenida). El trabajo no examinó por qué SRL-GAPA benefició más a los estudiantes con bajo nivel de SRL. No hay grupo control, sólo dos grupos donde se interviene con programas diferentes de promoción de SRL. En el estudio de Zambrano (2016), los autores indican que es una intervención piloto. Se aplicó a una muestra pequeña $(n=15)$ de estudiantes, por lo tanto los resultados no son extrapolables. Los instrumentos de medida del impacto del programa fueron indicadores de satisfacción. Se desconoce las competencias de autorregulación que mejoraron los alumnos, y cuánto mejoraron. El diseño del estudio no permite evaluar diferencias entre grupos, ya que no existe un grupo control y otro experimental. En el estudio de Rosário et al. (2016), las personas que aplicaron el programa de infusión curricular, fueron investigadores especialistas en autorregulación, y no docentes de una asignatura en el marco de un plan de estudio. En el estudio de Rodríguez y Martínez (2015), el diseño del estudio, no contempla un grupo control, por lo tanto, los resultados obtenidos, no se puede asegurar son efecto sólo de la intervención.

Respecto de las limitaciones asociadas al método, en el estudio de Ziegler y Moeller (2012), no se precisan cuáles son las variables dependientes del estudio, no se describen las propiedades psicométricas de los instrumentos, no se describen las características de los participantes, los mismos autores sugieren desarrollar una investigación más rigurosa para comprobar la causalidad entre el Linguafolio y el aumento de la autorregulación del aprendizaje. En el estudio de Santelices et al. (2014), no se midieron habilidades en los estudiantes, por lo tanto, se desconoce qué competencias de autorregulación mejoraron. En el estudio de Beaumont et al. (2016), el tamaño de la muestra y la restricción a un área temática única no permiten generalizar los resultados. En el estudio de Rodríguez y Martínez (2015), la muestra fueron estudiantes de tercer año de universidad. Lo recomendado por la literatura, es aplicar estos programas en primer año, ya que es cuando hay un menor desarrollo de la autorregulación en los estudiantes. El programa es descrito de forma general, no se precisa cada sesión desarrollada, los objetivos, las actividades, tiempo para cada una de ellas. Sólo se describe el tema a desarrollar por sesión. En el estudio de Parres y Flores (2011), una limitación fue la muestra utilizada, ya que los estudiantes tenían la creencia generalizada de que el arte es casi imposible aprenderlo si no cuenta con talento y habilidades innatas, así al ser invitados a participar en el estudio, se inscribieron sólo quienes previamente tenían cierto interés y curiosidad por el arte. Las creencias detectadas de las autoridades y profesores universitarios, sobre la concepción de que el arte es un mero entretenimiento y que no se relaciona con los contenidos temáticos de los programas educativos vigentes. En consecuencias a estas creencias, que implicó una muestra sesgada, no es posible replicar.

\section{Orientaciones de las intervenciones de tipo intracurricular}

Lin et al. (2016) sugieren un estudio longitudinal para saber: (a) cuándo deberían ser removidos los andamios externos (SRL-GAPA) para permitir a los estudiantes lograr la autorregulación, en lugar de co-regulación y, (b) cuánto tiempo dura la mejora de la SRL después del entrenamiento SRL-GAPA. También otro estudio para observar qué comportamientos se aprovechan de la conciencia de grupo y los mensajes interactivos entre sí. Conocer qué prácticas del profesor animan a los estudiantes al uso de estrategias, a observar el avance de comportamientos de aprendizaje de los compañeros en cada formación de SRL y a reflexionar sobre sus propias estrategias de aprendizaje.

Zambrano (2016), recomienda replicar experiencias de intervención pedagógica para promover el aprendizaje autorregulado en diferentes asignaturas y/o carreras, por la responsabilidad del docente universitario actual en promover en sus estudiantes además de los conocimientos propios de la disciplina que enseña, competencias necesarias para la vida. Ziegler y Moeller (2012), enfatizan la necesidad de intervenciones que contribuyan a la formación de docentes para que fomenten la autorregulación del aprendizaje e investigación sobre herramientas que permitan a los estudiantes autoevaluar su proceso de aprender. Beaumont et al. (2016), explican que existen principios sobre prácticas docentes en la evaluación y retroalimentación, pero no conceptualizan la retroalimentación sistemática dentro del proceso de evaluación, siendo responsabilidad del profesor intentar incorporarlos en su contexto particular. Es interesante y apropiado incorporar el diálogo iterativo, principalmente entre profesor y alumno en asignaturas de primer año para reforzar los principios del aprendizaje autorregulado, ayudando a los estudiantes a desarrollar las capacidades de autoevaluación considera esenciales. Sugieren incluir en futuras investigaciones retroalimentación con puntos de acción específicos para ayudar a los estudiantes a identificar cómo mejorar, incluyendo la enseñanza explícita de aspectos clave de la autorregulación, como el establecimiento de objetivos, la planificación, el seguimiento y la auto-instrucción.

Rosário et al. (2016) sugieren desarrollar programas que estimulen el desarrollo de competencias y procesos de aprendizaje autorregulado en Educación Superior, en formato de seminarios, dentro de las sesiones de 
bienvenida universitaria a los estudiantes de primer año o como asignaturas. Es necesario investigar la eficacia del programa a lo largo del tiempo, utilizando otras variables proceso (ej., diarios). Rodríguez y Martínez (2015), sugieren el desarrollo de programas de intervención para la promoción de la SRL donde se reporten los resultados y se establezcan las condiciones experimentales para su implementación. Esto debido a que en la literatura estas experiencias son escasas. Esta clase de programas en alumnos de ingeniería son particularmente importantes, como parte de la formación integral. Se debe entrenar a los profesores en el Coaching Educativo para desarrollar estos programas. Santelices et al. (2014), sugieren desarrollar un guion de instrucción para el profesor, donde el docente se orienta a estimular la autonomía del estudiante para enfrentar sus aprendizajes de manera guiada, ofreciendo un esquema y opciones de posibles tareas. Parres y Flores (2011), recomiendan la elaboración de un manual sobre estrategias de aprendizaje necesarias que el profesor promueva en sus estudiantes durante las clases, con formas particulares de participación tanto del profesor como de los estudiantes, con las que se propicie niveles cada vez más complejos de autorregulación y autonomía en el proceso de aprendizaje.

\section{DISCUSIÓN}

La presente investigación se planteó como objetivo caracterizar las intervenciones que desarrollan competencias de autorregulación y, describir, determinar limitaciones y orientaciones de intervenciones que la investigación señala necesaria implementar para la promoción de la autorregulación del aprendizaje. Las características de las intervenciones muestran diferentes formas para promover las estrategias de aprendizaje autorregulado en los estudiantes universitarios (Núñez, Solano, González-Pienda y Rosário, 2006). Principalmente existen dos modalidades para la aplicación de programas o intervenciones para la promoción de competencias de autorregulación, los programas extracurriculares y programas intra-curriculares (Zambrano, 2016). Los programas extracurriculares corresponden a cursos de capacitación, intervenciones puntuales o talleres (Núñez et al., 2006). Los programas intracurriculares, se caracterizan por la promoción de las competencias de autorregulación del aprendizaje en el contexto de un dominio específico (Zambrano, 2016), además del desarrollo del conocimiento de contenidos propios de una asignatura, el profesor promueve estrategias de disposición, cognitivas y metacognitivas en los estudiantes (Yáñez-Galleguillos y SoriaBarreto, 2017). Por lo tanto, un programa tiene la característica de intracurricular cuando es realizado en el plan de estudio (Pérez et al., 2011). Ya se ha dejado de lado el paradigma relacionado con completar lagunas de contenidos, siendo sustituido por la promoción de competencias para un aprendizaje autónomo (Rosário et al., 2007), sin embargo las referencias sobre eficacia de los programas intracurriculares de promoción de la autorregulación del aprendizaje aún son escasos en la literatura (Dresel et al., 2015; Rosário et al., 2014).

La presente investigación es coincide con los resultados de otros estudios que resaltan la necesidad de implementación de programas de intervención que muestren resultados empíricos y que además estas sean parte del currículum, pues aún se encuentra en un estado inicial de desarrollo (Cerezo et al., 2011; Rosário et al., 2014). Dada la escasez de trabajos validando la eficacia de programas intracurriculares de promoción de competencias y estrategias de autorregulación del aprendizaje, es comprensible la apelación de los investigadores, resaltando la necesidad de más investigaciones intracurriculares en educación centrada en la enseñanza intencional de estrategias de autorregulación (Dresel et al., 2015; Rosário et al., 2014; Zimmerman, 2008). En la presente investigación, 30 artículos cumplieron con los criterios de inclusión, 13 artículos corresponden a programas para el fomento de la autorregulación del aprendizaje, 5 de tipo extracurricular y 8 de tipo intracurricular. Los instrumentos de medición son todos de tipo autorreporte, sólo un programa utilizó medición procesual de las variables en estudio. La descripción de las ocho experiencias de programas intracurriculares muestran efectividad en la promoción de las competencias de autorregulación del aprendizaje. Las limitaciones de los programas se analizaron en su diseño, método y procedimiento. Respecto del diseño y método, las principales limitaciones fueron: inexistencia de grupo control para evaluar las diferencias entre grupos, muestra pequeña, muestra sesgada, instrumento de medida de satisfacción en vez de competencias de autorregulación, y no se precisan las variable dependientes. Respecto del procedimiento, las principales limitaciones fueron: falta de descripción del programa, inexistencia de validación de instrumentos o mención de sus propiedades psicométricas, y explicitar las consideraciones éticas.

Las orientaciones sugieren: programas longitudinales para evaluar la eficacia en el tiempo de los programas de intervención; programas que se apliquen en los primeros años de universidad, programas que reporten los resultados y se establezcan las condiciones experimentales para su implementación. Sin embargo, una orientación común de las investigaciones es la implicación de los profesores en el proceso de fomento de la autorregulación del aprendizaje. En el desarrollo de las tareas propias del currículum de su disciplina, los profesores pueden ofrecer modelos a sus alumnos y discutir con ellos la aplicación de estrategias de autorregulación a situaciones cotidianas de enseñanza-aprendizaje, facilitando primero su aplicación supervisada y, luego la aplicación autónoma (Pérez et al., 2010; Pérez et al., 2013). Para esto se proponen diferentes métodos como la formación o entrenamiento de docentes, un guion de instrucción, manual con estrategias que debe promover o actividades explícitas. 


\section{CONCLUSIONES}

Se puede concluir en la necesidad de (1) desarrollar programas de tipo intracurricular para la promoción de competencias de autorregulación del aprendizaje en estudiantes universitarios y (2) orientar nuevas investigaciones centradas en la implicación de los profesores en este proceso. Se justifica la implementación de programas de mejora de las estrategias de autorregulación con el fin de facilitar la transición de la Enseñanza Media a la Educación Superior en el currículo universitario de primer año (Vassallo, 2011; Beaumont et al., 2016), para contribuir a la formación integral de las nuevas generaciones de universitarios (Carbonero et al., 2013).

\section{AGRADECIMIENTOS}

Este artículo se realizó en el marco del proyecto de investigación "Impacto de un programa de entrenamiento en estrategias y creencias de disposición al estudio" para optar al grado de doctor en Psicología de la Universidad de Concepción, Chile. Cuenta con financiamiento de Becas Doctorado Nacional CONICYT Folio 21161224; y con apoyo del proyecto FONDECYT "Modelo explicativo de la permanencia y el abandono de los estudios universitarios, basado en procesos cognitivo motivacionales” № 1161502.

\section{REFERENCIAS}

Ato, M., J. López-García y A. Benavente, Un sistema de clasificación de los diseños de investigación en Psicología, doi: 10.6018/analesps.29.3.178511, Anales de Psicología, 29(3), 1038-1059 (2013)

Beaumont, C., S. Canning y C. Moscrop, Easing the transition from school to HE: scaffolding the development of selfregulated learning through a dialogic approach to feedback, doi: 10.1080/0309877X.2014.953460, Journal of Further and Higher Education, 40(3), 1-20 (2016)

Bembenutty, H., New directions for self-regulation of learning in postsecondary education, doi: 10.1002/tl.450, New Directions for teaching and Learning, 2016(126), 117-124 (2011)

Carbonero, M. y J. Navarro, Entrenamiento de alumnos de Educación Superior en estrategias de aprendizaje en matemáticas, Psicothema, 18(3), 348-352 (2006)

Carbonero, M., J. Román y M. Ferrer, Programa para aprender estratégicamente con estudiantes universitarios: diseño y validación experimental, doi: 10.6018/analesps.29.3.165671, Anales de Psicología, 29(3), 876-885 (2013)

Cassidy, S., Self-regulated Learning in Higher Education: Identifying key Component Processes, doi: 10.1080/03075079.2010.503269, Studies in Higher Education, 36(8), 989-1000 (2011)

Cazan, A., Self regulated learning strategies-predictors of academic adjustment, doi: 10.1016/j.sbspro.2012.01.092, Procedia-Social and Behavioral Sciences, 33, 104-108 (2012)

Cerezo, R., A. Bernardo y otros tres autores, Programas para la promoción de la autorregulación en educación superior: un estudio de la satisfacción diferencial entre metodología presencial y virtual, doi: 10.1016/j.ejeps.2015.10.004, European Journal of Education and Psychology, 8(1), 30-36 (2015)

Cerezo, R., J. Núñez y otros tres autores, Programas de intervención para la mejora de las competencias de aprendizaje autorregulado en educación superior, Perspectiva Educacional, 50(1), 1-30 (2011)

Cerezo, R., J. Núñez y otros tres autores, New media for the promotion self-regulated learning in higer education, Psicothema, 22(2), 306-315 (2010)

Chaves, E., J. Trujillo y J. López, Autorregulación del aprendizaje en entornos personales de aprendizaje en el Grado de Educación Primaria de la Universidad de Granada, España, doi:10.4067/S0718-50062015000400008, Formación Universitaria, 8(4), 63-76 (2015)

Cohen, M., The importance of self-regulation for college student learning, College Student Journal, 46(4), 892-902 (2012)

Dannefer, E., Beyond assessment of learning toward assessment for learning: Educating tomorrow's physicians, doi: 10.3109/0142159X.2013.787141, Medical Teacher, 35(7), 560-563 (2013)

De la Fuente, J., L. Zapata y otros cinco autores, Regulatory teaching and self-regulated learning in college students: confirmatory validation study of the IATLP scales, Electronic Journal of Research in Educational Psychology, 10(2), 839$866(2012)$

Da Silva, J. y E. Boruchovitch, Self-regulated learning in students of pedagogy, doi: 10.1590/198243272459201406, Paidéia, 24(59), 323-330 (2014)

Díaz, A., M. Pérez, J. González-Pienda y J. Núñez, Impacto de un entrenamiento en aprendizaje autorregulado en estudiantes universitarios, Perfiles Educativos, 39(157), 87-104 (2017)

Dörrenbächer, L. y F. Perels, More is more? Evaluation of interventions to foster self-regulated learning in college, International Journal of Educational Research, 78, 50-65 (2016) 
Dresel, M., B. Schmitz y otros nueve autores, Competencies for successful self-regulated learning in higher education: structural model and indications drawn from expert interviews, doi: 10.1016/j.ijer.2016.05.010, Studies in Higher Education, 40(3), 454-470 (2015)

Elvira-Valdés, M. y L. Pujol, Autorregulación y rendimiento académico en la transición secundaria-universidad, Revista Latinoamericana de Ciencias Sociales, Niñez y Juventud, 10(1), 367-378 (2012)

Fernández, E., A. Bernardo y otros cuatro autores, Predicción del uso de estrategias de autorregulación en educación superior, doi: 10.6018/analesps.29.3.139341, Anales de Psicología, 29(3), 865-875 (2013)

Gale, T. y S. Parker, Navigating change: a typology of student transition in higher education, Studies in Higher Education, 39(5), 734-753 (2014)

Garello, M. y M. Rinaudo, Autorregulación del aprendizaje, feedback y transferencia de conocimiento: Investigación de diseño con estudiantes universitarios, Revista Electrónica de Investigación Educativa, 15(2), 131-147 (2013)

Garello, M. y M. Rinaudo, Características de las tareas académicas que favorecen aprendizaje autorregulado y cognición distribuida en estudiantes universitarios, Revista de Docencia Universitaria, 10(3), 415-440 (2012)

Hernández, F., P. Rosário y J. Cuesta, Impacto de un programa de autorregulación del aprendizaje en estudiantes de Grado, Revista de Educación, (353), 71-588 (2010)

Houtman, E., Mind-Blowing: Fostering Self-Regulated Learning in Information Literacy Instruction, Communications in Information Literacy, 9(1), 6-18 (2015)

Hui, T. e I. Naufal, Does a Combination of Metaphor and Pairing Activity Help Programming Performance of Students with Different Self-Regulated Learning Level? Turkish Online Journal of Educational Technology, 10(4), 121-129 (2011)

Huie, F., A. Winsler y A. Kitsantas, Employment and first-year college achievement: the role of self-regulation and motivation, doi: 10.1080/13639080.2012.718746, Journal of Education and Work, 27(1), 110-135 (2014)

Kitsantas, A., Fostering college students' self-regulated learning with learning technologies, Hellenic Journal of Psychology, 10(3), 235-252 (2013)

Koivuniemi, M., E. Panadero, J. Malmberg y S. Järvelä, Higher education students' learning challenges and regulatory skills in different learning situations, Infancia y Aprendizaje, 40(1), 19-55 (2017)

Lam, R., Promoting self-regulated learning through portfolio assessment: testimony and recommendations, doi: 10.1080/02602938.2013.862211, Assessment and Evaluation in Higher Education, 39(39), 699-714 (2014)

Lin, J., Y. Lai. y L. Chang, Fostering self-regulated learning in a blended environment using group awareness and peer assistance as external scaffolds, doi: 10.1111/jcal.12120, Journal of Computer Assisted Learning, 32(1), 77-93 (2016)

Medrano, L., C. Galleano, M. Galera y F. Del Valle, Creencias irracionales, rendimiento y deserción académica en ingresantes universitarios, Liberabit, 16(2), 183-192 (2010)

Miranda, M. y J. Guzmán, Análisis de la Deserción de Estudiantes Universitarios usando Técnicas de Minería de Datos, doi:10.4067/S0718-50062017000300007, Formación Universitaria, 10(3), 61-68 (2017)

Montero, I. y O. León, A guide for naming research studies in Psychology, International Journal of Clinical and Health Psychology, 7(3), 847-862 (2007)

Navarrete, S., R. Candia y R. Puchi, Factores asociados a la deserción / retención de los estudiantes mapuche de la Universidad de la Frontera e incidencia de los programas de apoyo académico, doi: 10.4067/S0718-45652013000200003, Calidad en la Educación, (39), 43-80 (2013)

Núñez, J., R. Cerezo y otros cinco autores, Implementation of training programs in self-regulated learning strategies in Moodle format: Results of a experience in higher education, Psicothema, 23(2), 274-281 (2011)

Núñez, J., P. Solano, J. González-Pienda y P. Rosário, El aprendizaje autorregulado como medio y meta de la educación, Papeles del Psicólogo, 27(3), 139-146 (2006)

Panadero, E. y J. Alonso-Tapia, ¿Cómo autorregulan nuestros alumnos? Revisión del modelo cíclico de Zimmerman sobre autorregulación del aprendizaje, doi: 10.6018/analesps.30.2.167221, Anales de Psicología, 30(2), 450-462 (2014 a)

Panadero, E. y J. Alonso-Tapia, Teorías de autorregulación educativa: Una comparación y reflexión teórica, doi: 10.1016/j.pse.2014.05.002, Psicología Educativa, 20(1), 11-22 (2014 b)

Parres, R. y R. Flores, Experiencia educativa en arte visual diseñada bajo un modelo de autorregulación del aprendizaje con estudiantes universitarios, Revista Mexicana de Investigación Educativa, 16(49), 597-624 (2011)

Pérez, M., A. Díaz-Mujica, J. González-Pienda y J. Núñez, Autorregulación del aprendizaje en educación superior. En J. Catalán (Ed.), Psicología Educacional: proponiendo rumbos, problemáticas y aportaciones, pp. 49-79, Editorial de La Universidad de La Serena, Chile (2011)

Pérez, M., A. Díaz-Mujica, J. González-Pienda y J. Núñez, Docencia para facilitar el aprendizaje activo y autorregulado, Revista Diálogo Educacional, 10(30), 409-424 (2010)

Pérez, M., M. Valenzuela y otros tres autores, Disposición y enfoques de aprendizaje en estudiantes universitarios de primer año, Universitas Psychologica, 10(2), 441-449 (2011) 
Pérez, M., M. Valenzuela y otros tres autores, Dificultades de aprendizaje en estudiantes universitarios de primer año, doi: 10.4067/S0718-04622013000200010, Atenea (Concepción), 508, 135-150 (2013)

Pintrich, P., Multiple goals, multiple pathways: The role of goal orientation in learning and achievement, doi: 10.1037/00220663.92.3.544, Journal of Educational Psychology, 92(3), 544-555 (2000)

Rodríguez, L. y V. Martínez, Efectividad del coaching grupal sobre el desarrollo de la autorregulación del aprendizaje en estudiantes de ingeniería, Cuadernos de Investigación Educativa, 6(1), 71-88 (2015)

Rosário, P., S. Fuentes, M. Beuchat y A. Ramaciotti, Autorregulación del aprendizaje en una clase de la universidad: un enfoque de infusión curricular, doi: 10.6018/rie.34.1.229421, Revista de Investigación Educativa, 34(1), 31-49 (2016)

Rosário, P., R. Mourão y otros cuatro autores, Eficacia de un programa instruccional para la mejora de procesos y estrategias de aprendizaje en la enseñanza superior; Psicothema, 19(3), 422-427 (2007)

Rosário, P., J. Núñez y otros diez autores, Transcultural analysis of the effectiveness of a program to promote selfregulated learning in Mozambique, Chile, Portugal, and Spain, doi: 10.1080/07294360.2014.935932, Higher Education Research y Development, 34(1), 173-187 (2015)

Rosário, P., A. Pereira y otros seis autores, Autorregulación del aprendizaje: una revisión sistemática en revistas de la base SciELO, doi: 10.11144/Javeriana.UPSY13-2.aars, Universitas Psychologica, 13(2), 781-797 (2014)

Roux, R. y E. Anzures, Estrategias de aprendizaje y su relación con el rendimiento académico en estudiantes de una escuela privada de educación media superior; doi.org/10.15517/aie.v15i1.17731, Actualidades Investigativas en Educación, 15(1), 1-16 (2015)

Saíz, M., y A. Bol, Aprendizaje basado en la evaluación mediante rúbricas en educación superior, doi: 10.1016/S01214381(14)70004-9, Suma Psicológica, 21(1), 28-35 (2014)

Santelices, L., C. Williams, M. Soto y A. Dougnac, Efecto del enfoque de autorregulación del aprendizaje en la enseñanza de conceptos científicos en estudiantes universitarios en ciencias de la salud, Revista Médica de Chile, 142(3), 375-381 (2014)

Schober, B., J. Klug y otros seis autores, Gaining Substantial New Insights into University Students' Self-Regulated Learning Competencies, doi: 10.1027/2151-2604/a000201, Zeitschrift Für Psychologie, 223(1), 64-65 (2015)

Sitzmann, T. y K. Ely, A Meta-analysis of Self-regulated Learning in Work-related Training and Educational Attainment: What We Know and Where We Need to Go, doi: 10.1037/a0022777, Psychological Bulletin, 137(3), $421-540$ (2011)

Soto, V., Estudiantes de primera generación en Chile: una aproximación cualitativa a la experiencia universitaria, doi: 10.5209/rev_RCED.2016.v27.n3.47562, Revista Complutense de Educación, 27(3), 1157-1173 (2016)

Stoten, D., Managing the transition: a case study of selfregulation in the learning of first-term business and management undergraduate students at an English university, doi: 10.1080/13596748.2015.1081753, Research in Post-Compulsory Education, 20(4), 445-459 (2015)

Tuckman, B. y G. Kennedy, Teaching learning strategies to increase success of first-term college students, doi: 10.1080/00220973.2010.512318, The Journal of Experimental Education, 79(4), 478-504 (2011)

Vassallo, S., Implications of institutionalizing self-regulated learning: An analysis from four sociological perspectives, doi: 10.1080/00131946.2011.540984, Educational Studies, 47(1), 26-49 (2011)

Yáñez-Galleguillos, L. y K. Soria-Barreto, Reflexión de Buenas Prácticas Docentes como eje de Calidad en la Educación Universitaria: Caso Escuela de Ciencias Empresariales de la Universidad Católica del Norte, doi: 10.4067/S071850062017000500007, Formación Universitaria, 10(5), 59-68 (2017)

Yau, H., H. Sun y A. Fong, Adjusting to university: the Hong Kong experience, doi: 10.1080/1360080X.2012.642328, Journal of Higher Education Policy and Management, 34(1), 15-27 (2012)

Zambrano, C., Autoeficacia, Prácticas de Aprendizaje Autorregulado y Docencia para fomentar el Aprendizaje Autorregulado en un Curso de Ingeniería de Software, doi: 10.4067/S0718-50062016000300007, Formación Universitaria, 9 (3), 51-60 (2016)

Zarceño, A. y P. Andreu, Las tecnologías, un recurso didáctico que fortalece la autorregulación del aprendizaje en poblaciones excluidas, Perfiles Educativos, 37(148), 28-35 (2015)

Ziegler, N. y A. Moeller, Increasing Self-Regulated Learning through the LinguaFolio, doi: 10.1111/j.19449720.2012.01205.x, Foreign Language Annals, 45(3), 330-348 (2012)

Zimmerman, B., Investigating self-regulation and motivation: Historical background, methodological developments, and future prospects, doi: 10.3102/0002831207312909, American Educational Research Journal, 45(1), 166-183 (2008)

Zimmerman, B., Becoming a self-regulated learner: An overview. Theory in to Practice, doi: 10.1207/s15430421tip4102_2, 41(2), 64-70 (2002) 